Ephemeride des Kometen 1900 III (Giacobini) für die Erscheinung 1907. II. Teil. $\quad$ I $2^{\text {h }}$ M. Z. Berlin.

\begin{tabular}{|c|c|c|c|c|c|c|c|c|c|c|c|c|c|}
\hline \multirow[b]{2}{*}{1907} & \multicolumn{2}{|c|}{$x=-0.4$} & \multicolumn{2}{|c|}{$x=-0.2$} & \multicolumn{5}{|c|}{$x=0.0$} & \multicolumn{2}{|c|}{$x=+0.2$} & \multicolumn{2}{|c|}{$x=+0.4$} \\
\hline & $\alpha$ & 8 & $\alpha$ & 8 & $\alpha$ & 8 & $\log r$ & $\log \Delta$ & H & $\alpha$ & 8 & $x^{\prime} \mid$ & 8 \\
\hline März 21 & $2 \mathrm{I}^{\mathrm{h}} 5^{6^{\mathrm{m}}}$ & $+5: 6$ & $2 \mathrm{I}^{\mathrm{h}} 25^{\mathrm{m}}$ & $+5: 6$ & - & - & - & - & - & 一 & - & - & - \\
\hline 29 & $22 \quad 29$ & +7.4 & $21 \quad 55$ & +7.5 & - & - & - & - & - & - & - & - & - \\
\hline April 6 & 23 & +9.1 & 2228 & +9.5 & $2 \mathrm{I}^{\mathrm{h}} 53^{\mathrm{m}}$ & $+9: 6$ & 0.117 & 0.252 & O.I 5 & - & - & - & - \\
\hline I 4 & 2340 & +10.7 & 23 & $+\mathrm{II} .4$ & $22 \quad 25$ & $+\mathrm{r} 1.8$ & 0.092 & 0.235 & 0.18 & - & - & - & - \\
\hline 22 & $0 \quad 17$ & +12.0 & 2338 & $+13 \cdot 1$ & 2259 & +13.8 & 0.066 & $0.22 \mathrm{I}$ & 0.22 & $22^{\mathrm{h}} 25^{\mathrm{m}}$ & $+14: 3$ & - & - \\
\hline $3^{\circ}$ & 54 & +13.1 & - 1.5 & +14.5 & 2335 & +15.7 & 0.042 & 0.210 & 0.26 & $225^{8}$ & +16.6 & $22^{h} I^{m}$ & $+17: 2$ \\
\hline Iai & 33 & +13.8 & 54 & +15.5 & 012 & +17.2 & 0.018 & 0.203 & 0.30 & 2333 & +18.6 & 2244 & +19.9 \\
\hline 16 & $2 X I$ & +14.1 & 33 & +16.2 & $05 \mathrm{I}$ & +18.3 & 9.998 & 0.199 & 0.33 & 010 & +20.3 & 2320 & +22.3 \\
\hline
\end{tabular}

$$
\begin{aligned}
& \text { Für } x=-0.4 \text { ist } \mu=55^{\circ} \\
& x=-0.2 \text { " } \mu=547^{\prime \prime} \\
& x=0.0 \gg \mu=544^{\prime \prime} \\
& x=+0.2 \text { จ } \mu=54 \mathrm{I}^{\prime \prime} \\
& x=+0.4 \text { * } \mu=53^{\circ}
\end{aligned}
$$

Ekaterinoslaw, Höhere Bergschule, I 907 März I 2.
Vergleiche W. Abold und S. Scharbe: Definitive Bahn. bestimmung des Kometen I 900 III (Giacobini); Schriften der Naturforscher-Gesellschaft bei der Universität Jurjew -Dorpat.

\begin{tabular}{|c|c|c|c|c|}
\hline 1907 & $\alpha 1907.0$ & $\delta I 907.0$ & $\log r$ & $\log A$ \\
\hline Mărz 23 & I I ${ }^{\mathrm{h}} 29^{\mathrm{m}} 55^{\mathrm{s}}$ & $-5^{\circ} 55^{\prime} 7$ & 0.7140 & 0.6226 \\
\hline 27 & $\begin{array}{ll}27 & 57\end{array}$ & 545.2 & 7144 & 6239 \\
\hline $3^{I}$ & 264 & $5 \quad 34.5$ & 7147 & $625^{8}$ \\
\hline April 4 & $24 I 6$ & $\begin{array}{lll}5 & 23.9\end{array}$ & 7151 & 6281 \\
\hline 8 & 2233 & 5 I $3 \cdot 3$ & $7 \times 54$ & 6308 \\
\hline I 2 & 2059 & 53.0 & $715^{8}$ & 6340 \\
\hline I 6 & 1934 & $453 \cdot 1$ & 7162 & 6376 \\
\hline 20 & 1818 & $\begin{array}{ll}4 & 43.8\end{array}$ & 7166 & $64 \times 5$ \\
\hline 24 & 17 I 3 & $435 \cdot 1$ & 7169 & 6457 \\
\hline 28 & 1619 & 427.2 & 7 I 3 & 6502 \\
\hline Mai & I 535 & 420.1 & 7176 & $655^{\circ}$ \\
\hline 6 & I I I 53 & $-4 \times 3.9$ & $0.7+80$ & 0.6600 \\
\hline
\end{tabular}
Als Einheit der Helligkeit ist diejenige zur Zeit der

\begin{tabular}{|c|c|c|c|c|c|}
\hline \multicolumn{2}{|c|}{1907} & $\alpha 1907.0$ & $\delta$ I 907.0 & $\log r$ & $\log A$ \\
\hline Mai & 6 & $\operatorname{II}{ }^{h} 5^{m} 3^{s}$ & $-4^{\circ} \times 3^{\prime} \cdot 9$ & 0.7180 & 0.6600 \\
\hline & 10 & 1442 & $\begin{array}{ll}4 & 8.7\end{array}$ & 7183 & 6652 \\
\hline & I 4 & 1433 & $4 \quad 4 \cdot 5$ & 7187 & $67 \circ 5$ \\
\hline & 18 & 1436 & $4 \quad$ I. 3 & 7191 & 6760 \\
\hline & 22 & 1449 & 359.2 & 7194 & 68 I 5 \\
\hline & 26 & I $5 \quad 13$ & 358.3 & 7197 & $687 \mathrm{I}$ \\
\hline & 30 & I 547 & $3 \quad 5^{8.4}$ & 7201 & 6927 \\
\hline Juni & 3 & 1635 & 359.7 & 7205 & 6984 \\
\hline & 7 & 1731 & $4 \quad 2.0$ & 7208 & 7040 \\
\hline & I I & $18 \quad 37$ & $4 \quad 5 \cdot 4$ & 7211 & $7 \circ 96$ \\
\hline & 15 & 1952 & 410.0 & 7215 & 7151 \\
\hline & 19 & $\begin{array}{lll}\text { I I } & 21 & 17\end{array}$ & -4 I 5.6 & 0.7218 & 0.7205 \\
\hline
\end{tabular}
Entdeckung im Jahre I 900 angenommen worden.

\title{
Ephemeride des Planeten (588) [1906 TG].
}

$\mathrm{r}^{\mathrm{h}}$ M. Z. Berlin.

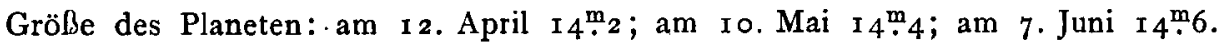

Diese Ephemeride ist eine Fortsetzung der in A. N. kanntgegebene Beobachtung, für welche jedoch der Ort des Nr. 4155 veröffentlichten. Ihr Fehler betrug bei der Wiederauffindung des Planeten durch Professor Wolf am 22. Januar $-46^{5}$ und $+5 \cdot 7$; eine von Prof. Barnard auf dem YerkesObservatory am 1 2. Februar angestellte und mir gütigst beVergleichsterns derzeit nur der Bonner Durchmusterung entnommen werden kann, gibt als Abweichung $-5 \mathrm{I}^{\mathrm{s}}$ und $+6: 7$. Es ist nicht wahrscheinlich, daß diese Beträge der Korrektionen bis zum Schluß der Ephemeride sehr anwachsen werden.

Triest, k. k. maritimes Observatorium, r9o7 März 9. F. Bidschof.

Inhalt zu Nr. 4163. E. Millosevich. Osservazioni di comete e pianetini. I6I. - Observations of Comet Finlay 1906 d. 167. - A. $\%$ Brunn. Beobachtungen von kleinen Planeten und Kometen. 169. - F. Frischauf. Zur Verläßlichkeit der 2I-stelligen Tafeln von Steinhauser. I73. - M. Giacobini. Eléments et éphéméride de la comète 1907 a. 173. - S. Scharbe. Ephemeride des Kometen 1900 III (Giacobini) für die Erscheịnung 1907. I75. - F. Bidschof. Ephemeride des Planeten (588) [1906 TG]. I75. - Berichtigungen. I75. 